\title{
Video Segmentation and Shot Boundary Detection Using Self-Organizing Maps
}

\author{
Hannes Muurinen and Jorma Laaksonen \\ Laboratory of Computer and Information Science* \\ Helsinki University of Technology \\ P.O. Box 5400, FIN-02015 TKK, Finland \\ \{hannes.muurinen, jorma. laaksonen\} @tkk.fi
}

\begin{abstract}
We present a video shot boundary detection (SBD) algorithm that spots discontinuities in visual stream by monitoring video frame trajectories on Self-Organizing Maps (SOMs). The SOM mapping compensates for the probability density differences in the feature space, and consequently distances between SOM coordinates are more informative than distances between plain feature vectors.

The proposed method compares two sliding best-matching unit windows instead of just measuring distances between two trajectory points, which increases the robustness of the detector. This can be seen as a variant of the adaptive threshold SBD methods. Furthermore, the robustness is increased by using a committee machine of multiple SOM-based detectors. Experimental evaluation made by NIST in the TRECVID evaluation confirms that the SOM-based SBD method works comparatively well in news video segmentation, especially in gradual transition detection.
\end{abstract}

Keywords: self-organizing map, video shot boundary detection.

\section{Introduction}

Consecutive frames within a shot, i.e. a continuous video segment that has been filmed in a single camera run, are usually visually similar. Shot boundaries on the other hand are characterised by discontinuities in the visual stream. Boundary detectors therefore often try to search for discontinuity peaks to spot boundaries. Various transition effects are available for video editors to bind shots together. The effects can be divided coarsely into abrupt cuts and gradual transitions based on their duration. In cut transitions there is an instantaneous change from one shot to another without any special effects, whereas in gradual transitions the shift has a nonzero duration, and there are transitional frames that do not belong exclusively to either one of the two shots.

Shot boundary detectors usually compute some distance measures between frames and the distances are compared to a threshold value to detect boundaries. The distance measures can be computed, for example, by comparing pixel

\footnotetext{
* Supported by the Academy of Finland in the projects Neural methods in information retrieval based on automatic content analysis and relevance feedback and Finnish Centre of Excellence in Adaptive Informatics Research.
} 
differences in consecutive motion-compensated frames, or by comparing some feature vectors or colour histograms calculated from the frames 1].

However, there can be differences of varying magnitude between consecutive frames within a shot, and the detector should be able to distinguish these from real shot boundaries. These differences are mainly caused by camera or object motion and lighting changes [2]. Especially camera flashlights are known to cause problems when handling news videos. A flashlight changes the illumination of the entire room for a short while, which is usually seen as two sharp discontinuity peaks in the visual flow. Also transmission errors might be seen as similar discontinuities. Ideally the detector should not react to this kind of differences, but only in the presence of a real boundary. In addition very slow gradual transitions induce further difficulties, since the detector should be sensitive enough to spot the slow transitions, but not too sensitive to react to fast visual changes within a shot. Adaptive threshold values have been used to resolve this challenge [3].

In this paper we will present a shot boundary detection method that compares two sliding frame windows on the Self-Organizing Map to spot the visual discontinuities. This can be seen as a variant of the adaptive threshold methods.

\section{Shot Boundary Detection by Trajectory Monitoring}

\subsection{Trajectories}

In our proposed technique the path that the data mapped on a Self-Organizing Map (SOM) 4] traverses as time advances is called a temporal trajectory. At each time step a feature vector is first calculated from the data, and then the vector is mapped to the best matching unit (BMU) on the map by finding the map node that has the most similar model vector. The process is illustrated in Figure 1] When visualising the trajectory, the consecutive BMUs are connected with line segments to form the full path as depicted in Figure 2 .

Feature extraction methods usually produce similar feature vectors for visually similar frames, and similar feature vectors are mapped to nearby regions on a Self-Organizing Map. Thus similar frames are mapped close to each other on the SOM, and distances between consecutive trajectory points can be used to spot discontinuities in the visual stream. Abrupt cuts are characterised by large leaps while gradual transitions can be seen as more gradual drifts from one map region to another. The drift pattern obviously depends on the feature extraction methods and map properties.

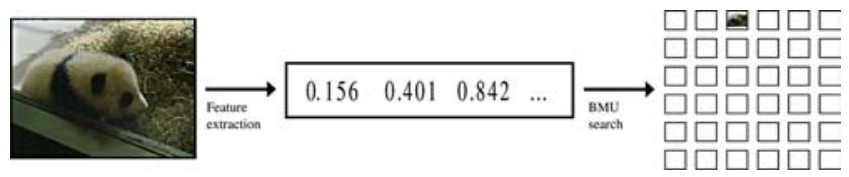

Fig. 1. A feature vector is calculated from the raw data. Then the vector is mapped on the SOM by finding the best-matching map unit. 

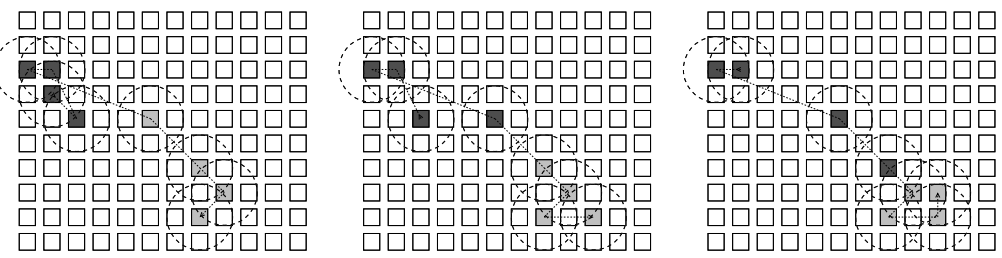

Fig. 2. Segments of a trajectory at three consecutive time steps. The SOM cells marked with dark gray colour represent trajectory points belonging to the set of preceding frames, and light gray cells represent the following frames. The trajectory is illustrated with a dotted black line, and the circles represent the area spanned by the preceding and following frame sets. The areas do not overlap in the two leftmost figures, but overlap at the third figure. This is interpreted as a one frame gradual transition.

\subsection{Sliding Frame Windows}

To increase the robustness of the proposed detector, distances between multiple frames before and after the current point of interest are compared instead of relying on map distances between two consecutive BMUs. Sliding frame windows on both sides of the point of interest are examined, and the corresponding map points on the trajectory are compared. The minimum distance between the map points of the two frame windows is identified and compared to a threshold value to determine the shot boundaries.

To express this formally, one has to define a distance measure that determines the distance between the two frame windows. If the lengths of the preceding and following frame windows are $l_{p}$ and $l_{f}$, the sets of the preceding and the following frames, $S_{p}(t)$ and $S_{f}(t)$, at time instants $t \in\left\{l_{p}, l_{p}+1, \ldots\right\}$ are

$$
\begin{aligned}
& S_{p}(t)=\left\{f_{t-l_{p}}, f_{t-l_{p}+1}, \ldots, f_{t-1}\right\} \\
& S_{f}(t)=\left\{f_{t}, f_{t+1}, \ldots, f_{t+l_{f}-1}\right\},
\end{aligned}
$$

where $f_{n}$ denotes frame $n$ of the video. The distance measured at time $t$ on SOM $k$ can then be formulated:

$$
d(k, t)=d\left(k ; S_{p}(t), S_{f}(t)\right)=\min _{f_{i}, f_{j}}\left\|C_{k}\left(f_{i}\right)-C_{k}\left(f_{j}\right)\right\| ; f_{i} \in S_{p}(t), f_{j} \in S_{f}(t) .
$$

Function $C_{k}\left(f_{n}\right)$ maps frame $f_{n}$ on SOM $k$ by calculating the corresponding map-specific feature vector value and finding the BMU. The function returns the discrete BMU coordinates $(x, y)$.

The distances between the frame windows are then compared to a fixed SOMspecific threshold $T_{k}$, and if $d(k, t)>T_{k}$, we declare that there is a shot boundary between the frame windows $S_{p}(t)$ and $S_{f}(t)$, i.e. between frames $f_{t-1}$ and $f_{t}$. Figure 2 illustrates how the preceding (dark gray) and following (light gray) frame windows are mapped on a SOM on one fictional trajectory at three consecutive time steps. The minimum distance of map coordinates between the two windows is then compared to the threshold value $T_{k}$ to detect shot boundaries. 
The method can also be described more intuitively by using the notion of areas. We may assume that separate shots occupy separate regions on the SOMs. A circular area that has radius of half the distance threshold value, $r_{k}=\frac{T_{k}}{2}$, is laid over each map point in the group of the preceding and following frames. When combined, the union of these areas form the spanned area of each frame window. If the two frame windows reside completely over separate shots, i.e. there is a boundary between the windows, the areas spanned by them do not overlap on the SOM. If the two windows contain frames that belong to the same shot, the areas probably do overlap. Therefore the separation of the areas can be used as an indicator of shot boundary locations. This is also illustrated in Figure 2. The BMU map points span an area around them, and the boundary classification can be done by checking if the circles around the light and dark gray map nodes overlap.

As mentioned, flashes and transmission error artifacts might cause sudden discontinuity peaks in the feature vector values. In a trajectory this can be seen as a jump to and back from some region on the SOM. If the lengths of the preceding and following frame windows are longer than the length of the distortion, the window method helps to prevent false positive detections during this kind of events.

Gradual transitions are characterised by multiple consecutive trajectory leaps. This kind of patterns can be detected using the same detector if the threshold values are suitably low. Consecutive boundary points are combined into a single data structure that specifies the boundary starting point and its length. Additionally we have defined a minimum length $L$ for the shots. If the detector has observed two boundaries that are too close to each other, these are combined into a single gradual transition. This is because shots with length of a couple of frames are not observable by humans, and the two separate boundary observations are clearly mistakes.

\subsection{Committee Machine of Detectors}

Some features might be better at detecting some type of transitions, while others might be better with other types. Therefore a committee machine of several parallel Self-Organizing Maps trained with different features is utilised in the detector. Additionally, a committee machine helps to reduce misclassifications if each detector does somewhat different mistakes, but they mostly agree on the correct boundary decisions.

The separate detectors run in parallel and return the locations of the detected transitions. The results of the committee are then combined. For each point between two consecutive frames $f_{t-1}$ and $f_{t}$ we check how the detectors have classified them. The final verdict is made by letting the detectors vote if a given point belongs into a transition. Each detector $k$ gives vote

$v_{k}(t)=\left\{\begin{array}{l}1 \text { if the detector determines that the point belongs into a transition, } \\ 0 \text { otherwise. }\end{array}\right.$ 
The weighted vote result is then computed as

$$
V(t)=\frac{\sum_{k=1}^{N} v_{k}(t) w_{k}}{\sum_{k=1}^{N} w_{k}},
$$

where $w_{k}$ is the weight of the $k$ th detector and $N$ is the total number of detectors. $V(t)$ is compared to a threshold value $T_{v} \in[0,1]$. If $V(t)>T_{v}$, we determine that the point between frames $f_{t-1}$ and $f_{t}$ is a boundary point. The transitions that are too close to each other are again combined, and the final starting locations and lengths of the transitions are obtained.

\subsection{Parameter Selection}

The shot boundary detection algorithm has quite a lot of parameters that can be tuned to change the behaviour of the detector. Some of the parameters are global, that is, they are shared by all the detectors in the committee, whereas some are detector-specific. Thus the number of free parameters increases as we increase the number of feature maps in the system. Table 1 summarises the global and map-specific parameters.

Some of the parameters were made detector-specific because optimal values for them are probably different for separate features. For example the distance threshold parameter, which determines the radius of the circles when comparing the areas spanned by the trajectory segments, should obviously be specific for each map because the lengths of significant jumps on trajectories might not be alike when using disparate features.

The parameter values were chosen using a variant of the discrete gradient descent method [5]. A set of training videos with human annotated shot boundary reference data were utilised in the parameter adjusting phase. The $F_{1}$ value [6] that combines precision $P$ and recall $R$ into a single scalar was used as the optimisation criterion. $F_{1}$ is a harmonic mean value that gives equal weight to both precision and recall, and it can be defined as

$$
F_{1}=\frac{2 P R}{P+R} .
$$

\section{$3 \quad$ Features}

Eleven feature extraction methods were applied to calculate feature vectors from all the frames in the videos. These were used to train feature-specific SOMs that

Table 1. The global and SOM-specific parameters that are freely selectable in the shot boundary detector. $k$ is the SOM index number.

\begin{tabular}{|l|l|}
\hline Global & Map-specific \\
\hline Preceding frame window length $l_{p}$ & Weight $w_{k}$ \\
Following frame window length $l_{f}$ & Distance threshold $T_{k}$ \\
Minimum shot length $L$ & \\
Vote result threshold $T_{v}$ & \\
\hline
\end{tabular}


were used in the detectors. Five of the features were standard MPEG-7 [7] descriptors: MPEG-7 Colour Structure, MPEG-7 Dominant Colour, MPEG-7 Scalable Colour, MPEG-7 Region Shape and MPEG-7 Edge Histogram. The remaining six features were Average Colour, Colour Moments, Texture Neighbourhood, Edge Histogram, Edge Co-occurrence and Edge Fourier. 8]

The Average Colour vector contains the average RGB values of all the pixels in the frame. The Colour Moments feature extractor separates the three colour channels of the HSV colour representation of the image. The first three moments, mean, variance and skewness, are estimated for each channel to create a ninedimensional feature vector.

The Texture Neighbourhood feature is calculated from the luminance component of the YIQ colour representation of an image. The 8-neighbourhood of each inner pixel is examined, and a probability estimate is calculated for the probabilities that the pixel in each of the surrounding relative position is brighter than the central pixel. The feature vector contains these eight estimates.

The Edge Histogram feature is not related to the MPEG-7 Edge Histogram descriptor. The feature vector consists of the histogram values of the four Sobel edge detectors. The Edge Co-occurrence vector contains values of the cooccurrence matrix calculated from the four Sobel edge detector outputs. The Edge Fourier feature vector contains 128 values computed from the Fast Fourier Transformation of the Sobel edge image of each frame. 9]

\section{Experiments}

\subsection{TRECVID Evaluation by NIST}

The performance of the shot boundary detector was evaluated by NIST in their annual TRECVID video retrieval evaluation, which consists of several video retrieval related tasks. In 2006 we participated in the shot boundary detection task for the first time 8]. The SBD task had 24 participating groups. Altogether there is slightly over 10 hours of news video in the SBD training and test data sets. The training data set also contains about 2.5 hours of NASA's educational programmes. The training files contain the total of 1341591 frames.

Ten separate detectors were trained using slightly differing portions of the training data and slightly differing combinations of feature extraction methods. The test data consisted of only news videos, and therefore we, for example, tested if omitting the NASA videos from the training set would improve the performance. Channel-specific detectors were also trained. We also tested if training detectors using only the cuts or only the gradual transitions would lead to better cut-specific and gradual-specific shot boundary detectors.

The best detector of our team turned out to be the one that had been trained with all the available data and with both cuts and gradual transitions. The cutspecific and gradual-specific detectors did not perform well. That is, when the detector had to make compromises between gradual and cut detection, its performance actually increased. This leads to suspicions of overlearning when using only a portion of the training data during the gradient descent optimisation. 
Our detectors performed comparatively well in the evaluation. Table 2 shows the performance of our best detectors compared to the TRECVID median and average. Separate $F_{1}$ values were calculated for cut and gradual transition detection, and additionally a total $F_{1}$ score combining these two was computed. For gradual transitions also frame recall, frame precision and frame $F_{1}$ values were computed. These describe how accurately the true starting and ending locations of the gradual transitions were found, whereas it is possible to get good gradual recall and precision even when there is only one frame overlapping between the reference and submitted transition windows. Frame precision and recall are calculated by comparing the number of frames that overlap in the submitted and reference transition windows to the total number of frames in these windows. A more comprehensive interpretation of the TRECVID results is given in 8 .

Table 2. The TRECVID shot boundary detection result comparison

\begin{tabular}{|l|l|l|l|l|}
\hline Detector & Total $F_{1}$ & Cut $F_{1}$ & Gradual $F_{1}$ & Frame $F_{1}$ \\
\hline Best single configuration & 0.709 & 0.732 & 0.647 & 0.716 \\
Best individual values out of ten & 0.709 & 0.732 & 0.654 & 0.737 \\
\hline TRECVID median & 0.747 & 0.792 & 0.625 & 0.734 \\
TRECVID average & 0.668 & 0.713 & 0.509 & 0.658 \\
TRECVID standard deviation & 0.199 & 0.214 & 0.250 & 0.212 \\
\hline
\end{tabular}

\subsection{Frame Window Experiment}

In addition to the TRECVID experiments three detector configurations were compared to investigate how much the detector benefited from using frame windows instead of single frames in distance computations. The parameters of the first detector were optimised using all the training data, both cuts and gradual transitions, and the following six feature maps: Average Colour, Colour Moments, Texture Neighbourhood, Edge Histogram, MPEG-7 Dominant Colour and MPEG-7 Colour Structure. These features obtained the highest weights $w_{k}$ in the TRECVID experiment. All the parameters including the window lengths were freely adjustable. The second detector was trained using the same features and training data, but we fixed the window lengths to $l_{p}=l_{f}=1$. We also tested an unoptimised version of the one-frame detector by using the optimal parameters of the first configuration and just setting the window lengths to one.

Figure 3 depicts the precision and recall values of the three detectors. By comparing the performance values we can confirm that the frame windows contribute significantly to the overall performance of the detector. Rather good results could be obtained also using the optimised one-frame detector.

We can interpret the result of this experiment by referring to the idea of areas spanned by the frame windows. The optimised one-frame detector tries to model the area occupied by the given shot using the map location of only one frame. With a suitably large circle radius this can be harshly approximated, but the detector that models the area using multiple sequential map coordinates and the union of smaller circles can represent this area much more accurately. 

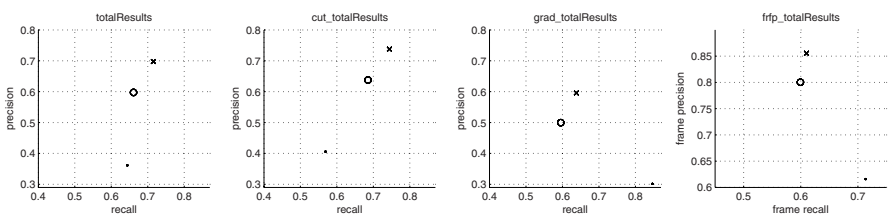

Fig. 3. The performance values of the detector with freely adjustable window lengths (cross), and the optimised (circle) and unoptimised (dot) detectors with window length of one

Comparing frame windows instead of distances between two frames can be also seen as a variant of the adaptive distance threshold methods. In fast-paced shots the area spanned by the frame window is much larger than during a slowpaced shot. This is comparable to the growth of an adaptive threshold value during content with high motion activity. If the frame window length is fixed to one, the distance threshold is constant. It can be argued that our method can be more accurate than using an adaptive one-dimensional threshold value. This is because in our method the adaptive threshold value is not a scalar that increases the threshold equally to all directions on the map, but there is an adaptively transforming two-dimensional threshold boundary that propagates selectively towards specific directions on the map.

\subsection{Feature Number Experiment}

In our last experiment twelve detectors using varying number of features were compared. First a detector with all the eleven features was trained, and then the number of features was decreased by discarding the feature that got the lowest weight $w_{k}$ in the previous training run. Two one-feature detectors were also tested to compare the performance of the best edge feature to the performance of the best colour feature. The features were in the order of importance: Edge Histogram, Average Colour, Colour Moments, MPEG-7 Dominant Colour, Texture Neighbourhood, MPEG-7 Colour Structure, MPEG-7 Region Shape, MPEG-7 Edge Histogram, MPEG-7 Scalable Colour, Edge Co-occurrence and Edge Fourier.

The precision and recall values for the detectors with varying number of features are shown in Figure 4. It seems like the overall performance increases as the number of features increases. This was expected, although we anticipated overlearning when the number of features increased too much. This does not seem to happen with the maximum number of eleven features we have used. Using both cuts and gradual transitions in training may be one reason why the overlearning problem has not manifested itself.

The single colour feature seemed to be better in gradual transition detection than the single edge feature. This can be seen also in Figure 5 that shows a more detailed experiment in which the distance thresholds $T_{k}$ were varied for the two one-feature detectors. Combining the two features clearly improves the results, which was expected because the amount of information available to the 

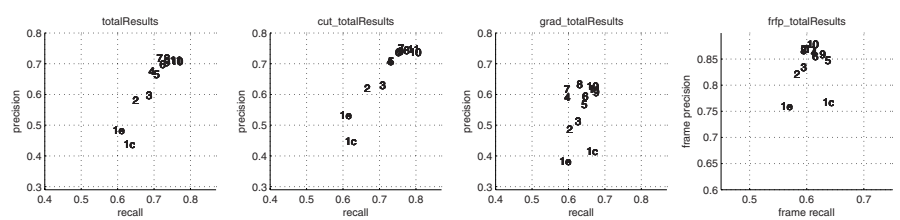

Fig. 4. Recall and precision for detectors using varying number of features. Detector $1 \mathrm{e}$ is a one feature detector using the Edge Histogram feature and detector $1 \mathrm{c}$ is a detector using the Average Colour feature.
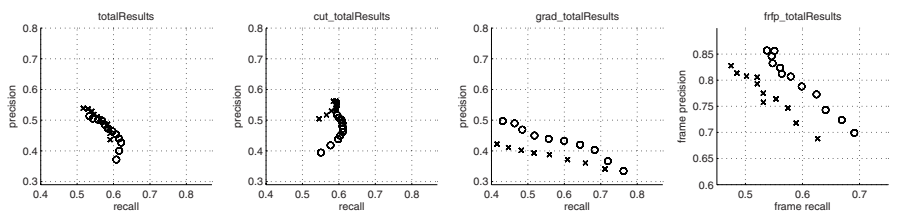

Fig. 5. Recall and precision for Average Colour (circle) and Edge Histogram (cross) based detectors with varying distance threshold parameters

detector increased. The cut detection performance values of the two detectors are similar. The edge feature configuration has better precision, while the colour feature configuration has slightly better recall.

\section{Conclusions}

A shot boundary detector using parallel Self-Organizing Maps was implemented during this work. We used it to participate in TRECVID shot boundary detection task, and our SOM-based method seemed to work quite well. Gradual transition detection performance was above the TRECVID median and cut detection performance above the TRECVID average.

The proposed SBD method tries to spot discontinuities in SOM trajectories to detect transitions. The novel idea is to use frame windows and areas spanned by the frame windows in detection. The areas occupied by preceding and following frame windows are approximated by using a union of circles centred on the map points corresponding to the frames. We assume that separate shots occupy separate regions on the map. If the areas occupied by the two frame windows do not overlap, they clearly belong to distinct shots, and a shot boundary between the frame windows can be declared. In the experiments this approach proved to be clearly better than the alternative method of just comparing the distances of two map points on the trajectory.

The feature vectors calculated from the frames could have been used on their own to detect shot boundaries, but in the implemented detector an additional step of mapping the vectors on a SOM was performed before measuring the distances between the frames. The transitions on a SOM are more informative 
than the distances between feature vectors because the nonlinear mapping of the SOM algorithm compensates the probability density differences in feature space, i.e the SOM takes into account that some regions in the feature space might be more probable than others.

Furthermore, in this algorithm the vector quantisation property of SOMs is helpful as it helps to suppress the little variations between the visually similar frames within a shot. Only significantly large changes in the input vectors are seen as movement on the maps. In general vector quantisation can cause problems since in the worst case scenario two vectors can be mapped to neighbouring nodes even when they reside right next to each other in the vector space. In practice this does not cause problems in our system because the distance threshold parameters always converged to such values that trajectory drifts to neighbouring SOM nodes are not interpreted as shot transitions.

The feature extraction methods utilised in our detector have been originally developed for image and video retrieval, and they might not be optimal in shot boundary detection task. Developing new SBD-optimised feature extraction methods might increase the performance of the detector. Especially lighting, translation and rotation invariance would be desired properties in shot boundary detection. Furthermore, new objects arriving from the edges of the screen should not change the feature vector values too much. Perhaps this could be achieved by using feature extraction methods that give gradually diminishing amount of weight to the pixels near the edges of the image.

\section{References}

1. Rui, Y., Huang, T.S., Mehrotra, S.: Exploring video structure beyond the shots. In: International Conference on Multimedia Computing and Systems. pp. 237-240 (1998)

2. Hanjalic, A.: Shot-boundary detection: unraveled and resolved? IEEE Trans. Circuits Syst. Video Techn. 12(2), 90-105 (2002)

3. Yeo, B.L., Liu, B.: Rapid scene analysis on compressed video. Circuits and Systems for Video Technology, IEEE Transactions 5(6), 533-544 (1995)

4. Kohonen, T.: Self-Organizing Maps, 3rd edn. Springer Series in Information Sciences. Springer, Heidelberg (2001)

5. Christensen, J., Marks, J., Shieber, S.: An empirical study of algorithms for pointfeature label placement. ACM Trans. Graph. 14(3), 203-232 (1995)

6. Van Rijsbergen, C.J.: Information Retrieval, 2nd edn. Dept. of Computer Science, University of Glasgow (1979)

7. ISO/IEC: Information technology - Multimedia content description interface - Part 3: Visual, 15938-3:2002(E) (2002)

8. Sjöberg, M., Muurinen, H., Laaksonen, J., Koskela, M.: PicSOM experiments in TRECVID 2006. In: Proceedings of the TRECVID 2006 Workshop, Gaithersburg, MD, USA (November 2006)

9. Brandt, S., Laaksonen, J., Oja, E.: Statistical shape features for content-based image retrieval. Journal of Mathematical Imaging and Vision 17(2), 187-198 (2002) 